\title{
FORMS OF VERITABLE AND RELATIVE TRAJECTORIES OF DIVERGENCE OF SHIP
}

\section{ФОРМЫ ИСТИННОЙ И ОТНОСИТЕЛЬНОЙ ТРАЕКТОРИЙ РАСХОЖДЕНИЯ СУДНА}

\author{
T.Y. Omelchenko, senior lecturer, V.V. Kaliuzhniy, PhD student, \\ A.V. Borodulin, $P h D$ student
}

Т.Ю. Омельченко, старший преподаватель, В.В. Калюжный, аспирант,

А.В. Бородулин, аспирант

National University «Odessa Maritime Academy», Ukraine

Национальный университет «Одесская морская академия», Украина

\begin{abstract}
The forms of veritable and relative trajectories of divergence are considered. It is shown that at speed of ship of greater speed the whole forms of veritable and relative trajectories coincide. For a case, when speed of ship less speed of target certain dependence of form of relative trajectory on the form of veritable trajectory.

So as the forms of veritable trajectory get out for divergence, and their parameters depend on the form of relative trajectory of divergence, communication of forms of relative and veritable trajectories of divergence is considered. It is shown that two forms of veritable trajectory of standard strategy of divergence are and four forms of relative trajectory of divergence can answer them in general case.

Therefore, in work the reflection of two elementary forms of veritable trajectory of divergence of ship is considered deviation to the right and to the left in the great number of forms of relative trajectory of divergence.

If speed of ship more large to speed of target, regardless of side of veritable deviation the forms of veritable and relative trajectories coincide, that direction of veritable deviation, as well as direction of veritable output on a previous course coincide with the proper relative directions. It is conditioned proportional dependence of relative course on a veritable course for the case of speed of ship of target larger from speed.

In time, when speed of ship more small to speed of target, dependence of relative course on a veritable course has both the intervals of growth and intervals of falling. In this communication accordance disappears between a relative course and veritable course. Therefore, can answer the interval of growth of veritable course, as interval of growth, so interval of falling of relative course. It is just for the cases of veritable deviation, both to the right and to the left, that shows the ambiguous reflection of two forms of veritable trajectory of divergence of ship in the great number of four forms.

In the article four examples of reflection of form of veritable trajectory of divergence by deviation are resulted to the right in each of forms of relative trajectory of divergence.
\end{abstract}


Similar results are got for the elementary form of veritable trajectory of divergence of ship deviation of course to the left. It is shown in both cases, that size of interval of growth, as well as the interval of falling of relative course is determined by the relation of speeds of ship and target.

Keywords: safety of navigation, warning of collision of vessels, form of veritable and relative trajectories of divergence.

\section{PEФЕРАT}

Розглянуто форми істинної та відносної траєкторій розходження. Показано, що при швидкості судна більшої швидкості цілі форми істинної та відносної траєкторій співпадають. Для випадку, коли швидкість судна менше швидкості цілі визначена залежність форми відносної траєкторії від форми істинної траєкторії.

Так як для розходження вибираються форми істинної траєкторії, а їх параметри залежать від форми відносної траєкторії розходження, то розглянуто зв'язок форм відносної і істинної траєкторій розходження. Показано, що існує дві форми істинної траєкторії стандартної стратегії розходження і в загальному випадку їм можуть відповідати чотири форми відносної траєкторії розходження.

Тому в роботі розглянуто відображення двох елементарних форм істинної траєкторії розходження судна ухиленням вправо та вліво в множину форм відносної траєкторії розходження.

Якщо швидкість судна більша швидкості цілі, то незалежно від сторони істинного ухилення форми істинної та відносної траєкторій співпадають, тобто напрям істинного ухилення, як і напрям істинного виходу на попередній курс співпадають 3 відповідними відносними напрямами. Це обумовлено пропорційною залежність відносного курсу від істинного курсу для випадку швидкості судна більшої від швидкості цілі.

В разі, коли швидкість судна менша швидкості цілі, залежність відносного курсу від істинного курсу має як інтервали зростання, так і інтервали падіння. В зв'язку з цим відповідність між відносним курсом і істинним курсом зникає. Тому інтервалу зростання істинного курсу може відповідати, як інтервал зростання, так інтервал падіння відносного курсу. Це справедливо для випадків істинного ухилення, як вправо, так i вліво, що показує неоднозначне відображення двох форм істинної траєкторії розходження судна в множину чотирьох форм.

В статті приведено чотири приклади відображення форми істинної траєкторії розходження ухиленням вправо в кожну із форм відносної траєкторії розходження.

Аналогічні результати отримані для елементарної форми істинної траєкторії розходження судна ухиленням курсу вліво. В обох випадках показано, що величина інтервалу зростання, як і інтервалу падіння відносного курсу визначається відношенням швидкостей судна і цілі. 
Ключові слова: безпека судноводіння, попередження зіткнень суден, форми істинної та відносної траєкторій розходження.

\section{Постановка проблемы в общем виде и ее связь с важными научными или практическими задачами}

Важнейшей проблемой обеспечения безопасности судовождения является снижение аварийности судов при плавании в стесненных водах. Аварийность по причине столкновений судов до сих пор находится на высоком уровне, что обуславливает необходимость разработки эффективных мер по предупреждению их столкновений.

Следовательно, разработка способов совершенствования процесса расхождения опасно сближающихся судов, в частности учетом относительной формы траектории расхождения для расчета параметров стратегии расхождения судна и зависимости относительной формы расхождения от истинной траектории, чему посвящена данная статья, является актуальным и перспективным научным направлением.

Анализ последних достижений и публикаций, в которых начато решение данной проблемы и выделение нерешенных ранее частей общей проблемы

Основные вопросы предупреждения столкновения судов освещены в работах [1] и [2], а работа [3] посвящена формированию безопасных доменов. Работа [4] рассматривает выбор ситуации сближения судна с целью из множества стандартных ситуаций, после чего производится определение стратегии расхождения. При возникновении ситуации чрезмерного сближения судов в работе [5] предлагается способ формирования стратегии экстренного расхождения.

\section{Формулировка целей статьи (постановка задачи)}

Целью статьи является установление зависимости формы относительной траектории расхождения от истинной траектории в случае, когда скорость судна меньше скорости цели.

Изложение основного материала исследования с обоснованием полученных научных результатов

Для корректных расчетов параметров маневра расхождения оперирующего судна, опасно сближающегося с целью необходимо рассмотреть формы истинной и относительной траекторий расхождения, a также их взаимозависимость.

Форма истинной траектории расхождения, которая характеризуется уклонением вправо $\delta_{\text {tst }}$ показана на рис. 1, а форма истинной траектории расхождения уклонением влево обозначена $\delta_{\text {tpr }}$ и показана на том же рисунке. 


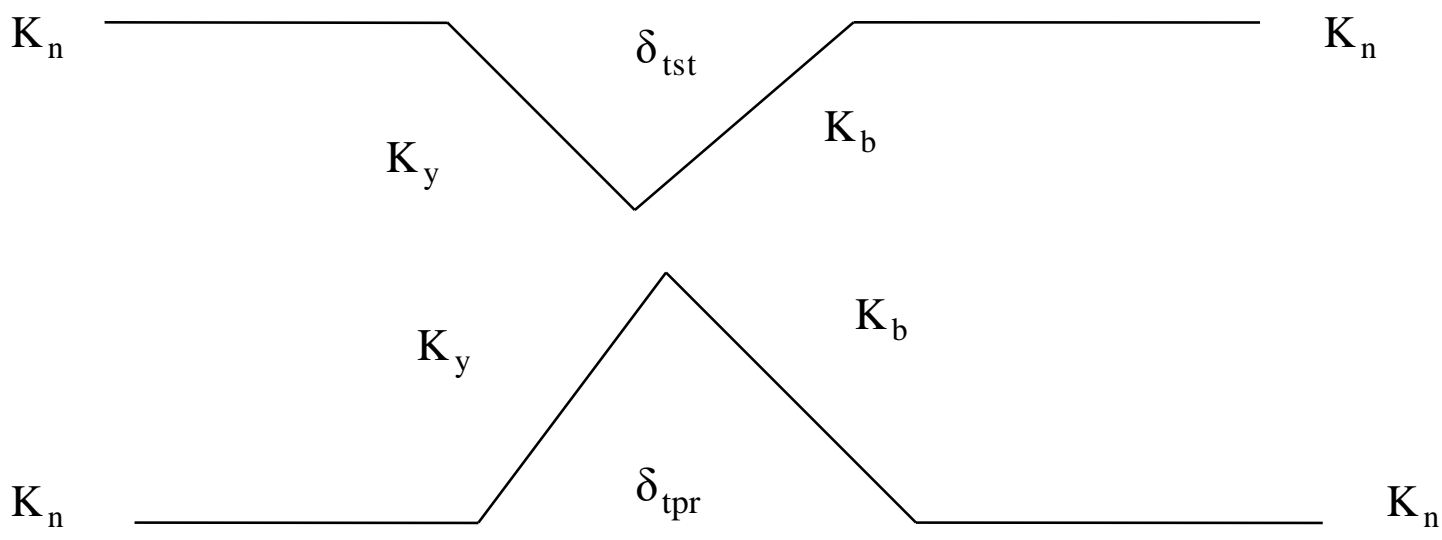

Рис. 1. Формы истинной траектории расхождения

В случае, когда маневр расхождения выполняется судном, скорость которого больше скорости цели, т. е. $\mathrm{V}_{\mathrm{o}}>\mathrm{V}_{\mathrm{c}}$, то формы истинной и относительной траекторий совпадают. Обозначим относительную траекторию, у которой относительный курс уклонения больше начального относительного курса, $\mathrm{K}_{\text {oty }}>\mathrm{K}_{\text {otn }}$ и $\mathrm{K}_{\text {otb }}<\mathrm{K}_{\text {oty }}$, через $\Delta_{\text {tst }}$ (рис. 2). Относительная форма $\Delta_{\text {tpr }}$, для которой характерны соотношения $\mathrm{K}_{\text {oty }}<\mathrm{K}_{\text {otn }}$ и $\mathrm{K}_{\text {otb }}>\mathrm{K}_{\text {oty }}$, как показано также на рис. 2.

В дальнейшем рассмотрим ситуацию, когда скорость маневрирующего судна меньше скорости цели, т. е. $\mathrm{V}_{\mathrm{o}}<\mathrm{V}_{\mathrm{c}}$. При этом необходимо учитывать, что при увеличении истинного курса судна $\mathrm{K}_{\mathrm{o}}$ относительный курс $\mathrm{K}_{\text {ot }}$ имеет как участок роста, так и участок убывания. На рис. 3 показана ситуация, когда начальный курс судна $\mathrm{K}_{\mathrm{n}}$ и курсы уклонения $\mathrm{K}_{\mathrm{y}}$ и возвращения на заданную траекторию $\mathrm{K}_{\mathrm{b}}$ находятся на растущем участке зависимости относительного курса. В этом случае форме $\delta_{\text {tst }}$ истинной траектории соответствует форма $\Delta_{\text {tst }}$ относительной траектории, т. е. $\delta_{\text {tst }} \rightarrow \Delta_{\text {tst }}$. Если же курсы $\mathrm{K}_{\mathrm{n}}, \mathrm{K}_{\mathrm{y}}$ и $\mathrm{K}_{\mathrm{b}}$ формы истинной траектории расхождения $\delta_{\text {tst }}$ принадлежат убывающему участку зависимости относительного курса, как показано на рис. 4, то форма относительной траектории расхождения не совпадает с формой истинной траектории, а имеет место соответствие $\delta_{\mathrm{tst}} \rightarrow \Delta_{\mathrm{tpr}}$. В дальнейшем рассмотрим ситуации, когда курсы истинной траектории расхождения находятся на разных участках зависимости относительного курса. 


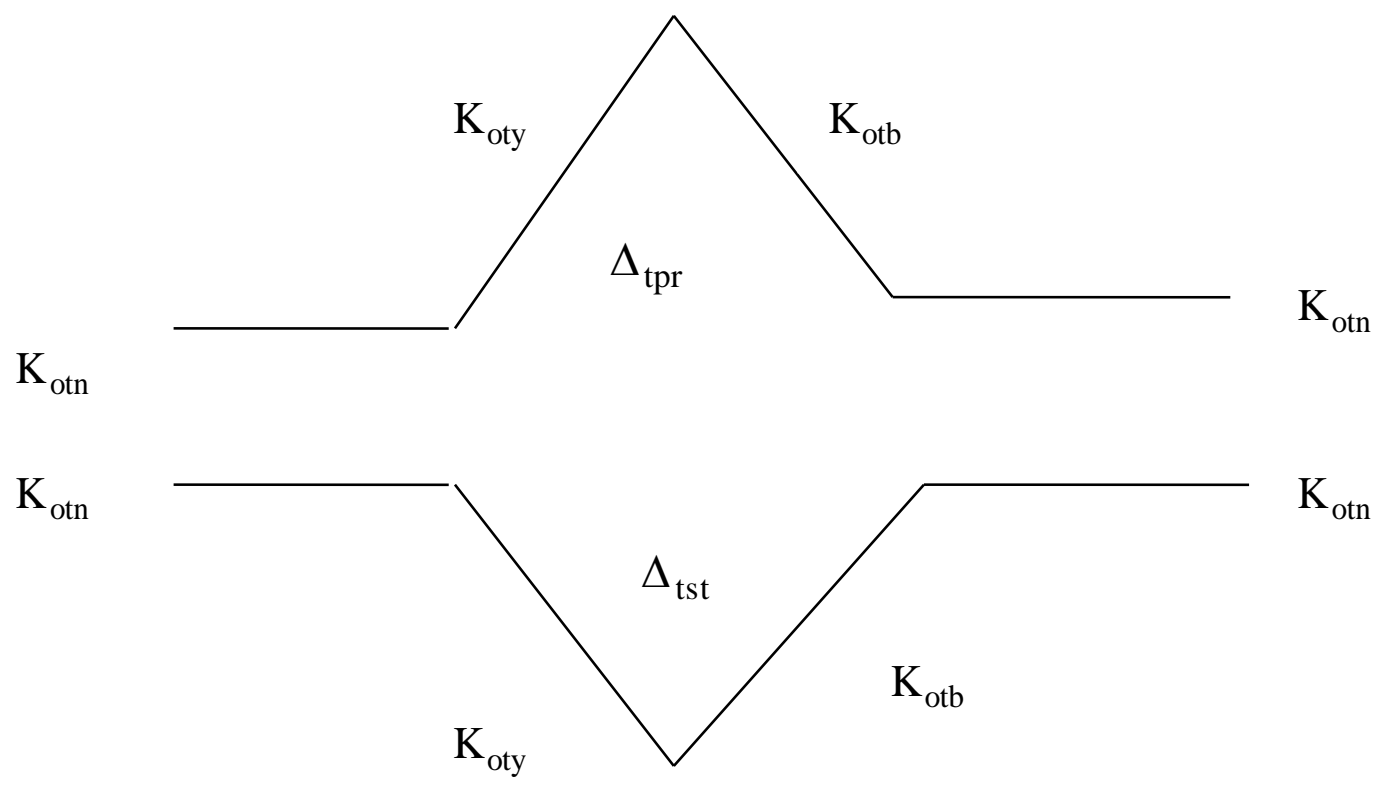

Рис. 2. Формы относительной траектории расхождения $\Delta_{\mathrm{tst}} u \Delta_{\mathrm{tpr}}$
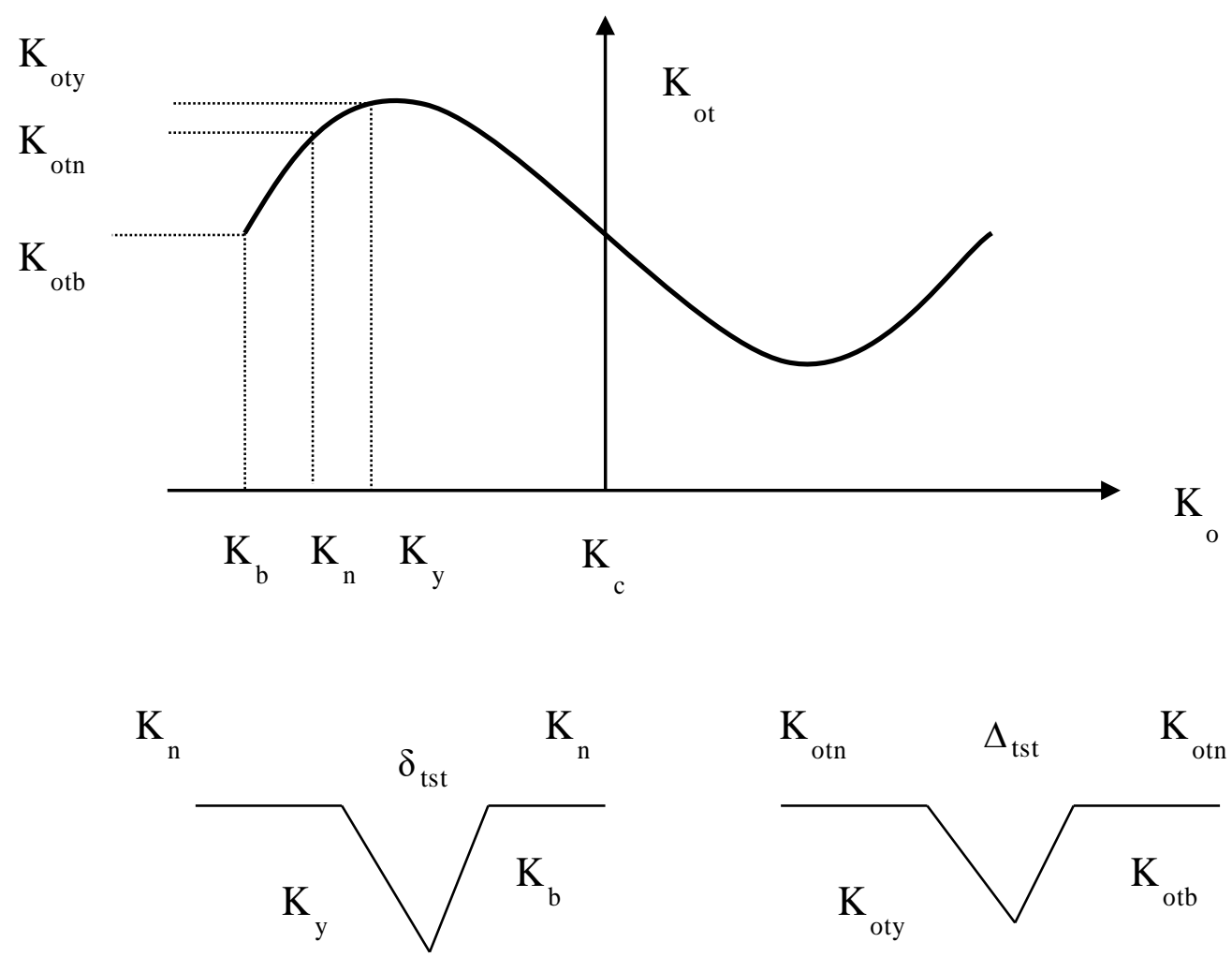

Pис. 3. Соответствие $\delta_{\mathrm{tst}} \rightarrow \Delta_{\mathrm{tst}}$ форм траекторий при $\mathrm{V}_{\mathrm{o}}<\mathrm{V}_{\mathrm{c}}$

Вначале допустим, что курсы $\mathrm{K}_{\mathrm{n}}$ и $\mathrm{K}_{\mathrm{b}}$ формы $\delta_{\text {tst }}$ истинной траектории принадлежат растущему участку зависимости относительного курса, а курс уклонения $\mathrm{K}_{\text {y }}$ - убывающему участку, как показано на рис. 5.В показанном 
примере относительная траектория имеет участки уклонения и выхода, которые изменяются влево. Такую форму траектории расхождения обозначим $\Delta_{\operatorname{tpr} 1}$. Соответствие $\delta_{\text {tst }} \rightarrow \Delta_{\text {tpr1 }}$ истинной и относительных траекторий показано на рис. 5.

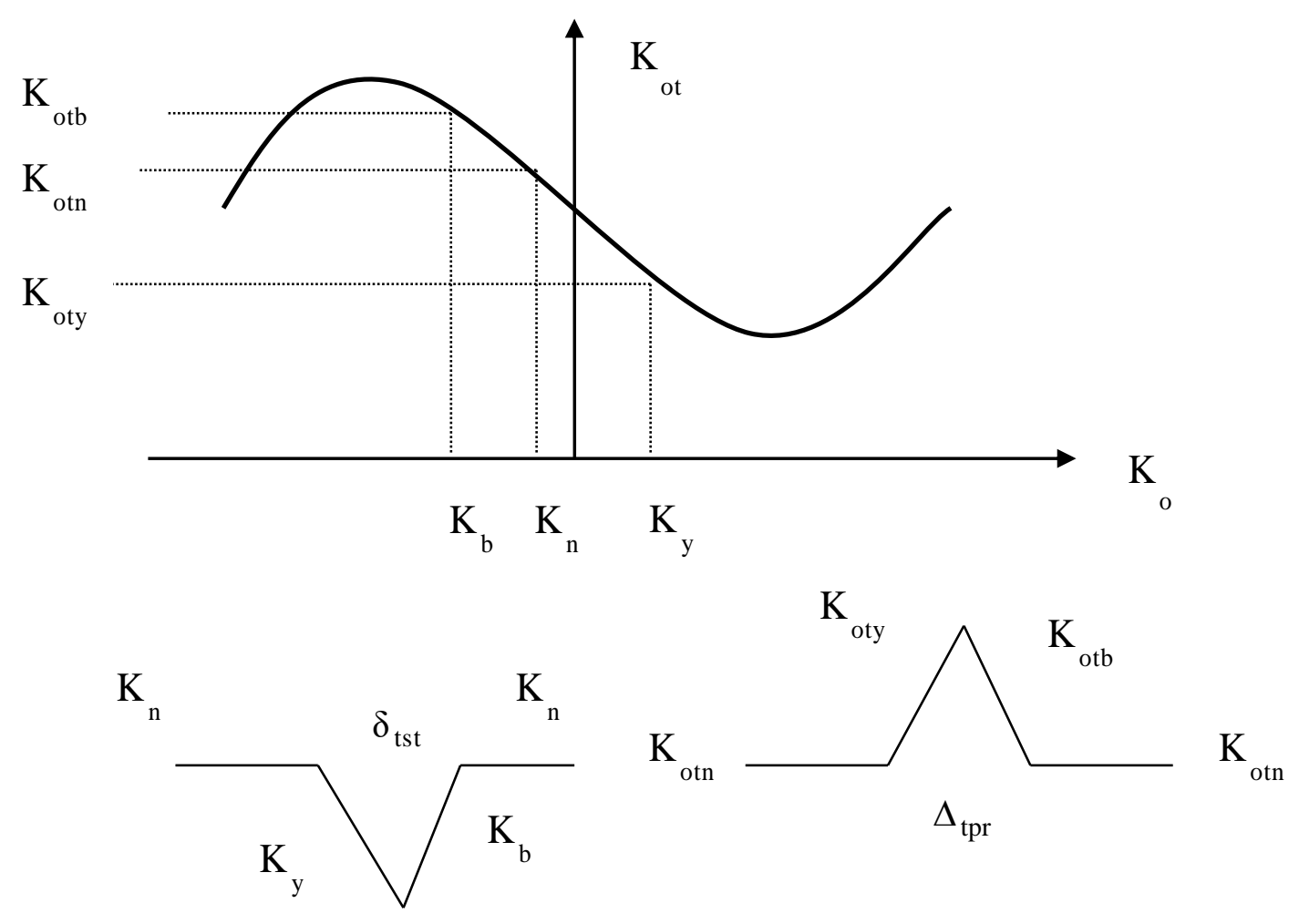

Рис. 4. Соответствие $\delta_{\text {tst }} \rightarrow \Delta_{\text {tpr }}$ форм траекторий при $\mathrm{V}_{\mathrm{o}}<\mathrm{V}_{\mathrm{c}}$

На рис. 6 курсы $\mathrm{K}_{\mathrm{n}}$ и $\mathrm{K}_{\mathrm{b}}$ формы $\delta_{\text {tst }}$ истинной траектории принадлежат убывающему участку зависимости относительного курса, а курс уклонения $\mathrm{K}_{\mathrm{y}}$ - растущему участку. Относительная траектория, соответствующая этому случаю, характеризуется следующей особенностью: ее участки уклонения и выхода на заданную траекторию изменяются вправо по отношению к предыдущему участку. Такую форму относительной траектории с двумя участками изменения курса вправо обозначим через $\Delta_{\text {tst1 }}$, причем можно утверждать, что существуют соотношения курсов $\mathrm{K}_{\mathrm{n}}, \mathrm{K}_{\mathrm{y}}$ и $\mathrm{K}_{\mathrm{b}}$ формы $\delta_{\text {tst }}$ истинной траектории расхождения, при которых имеет место соответствие $\delta_{\text {tst }} \rightarrow \Delta_{\text {tst1 }}$ (рис. 6). 


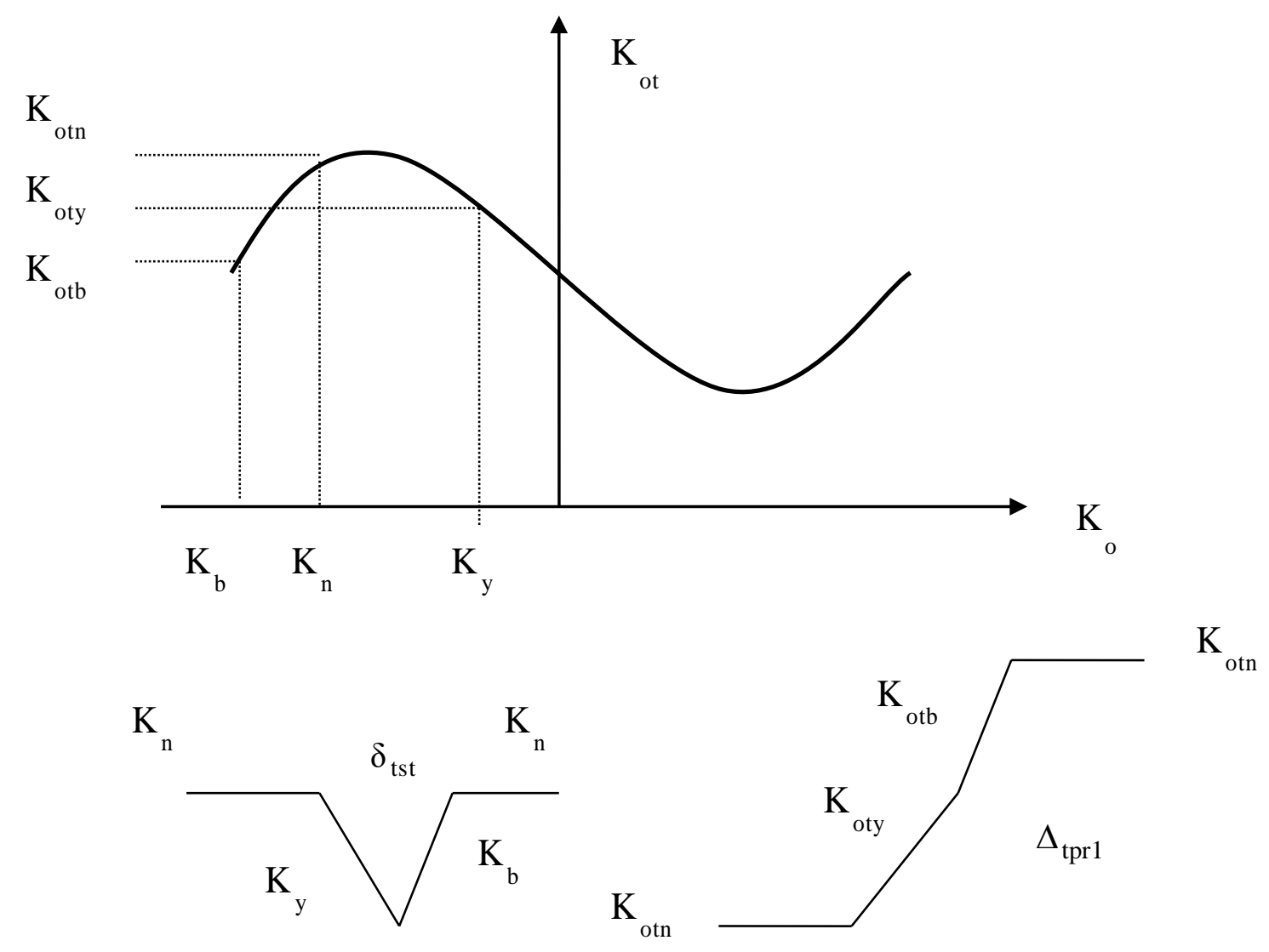

Pис. 5. Соответствие $\delta_{\mathrm{tst}} \rightarrow \Delta_{\mathrm{tpr} 1}$ форм траекторий при $\mathrm{V}_{\mathrm{o}}<\mathrm{V}_{\mathrm{c}}$
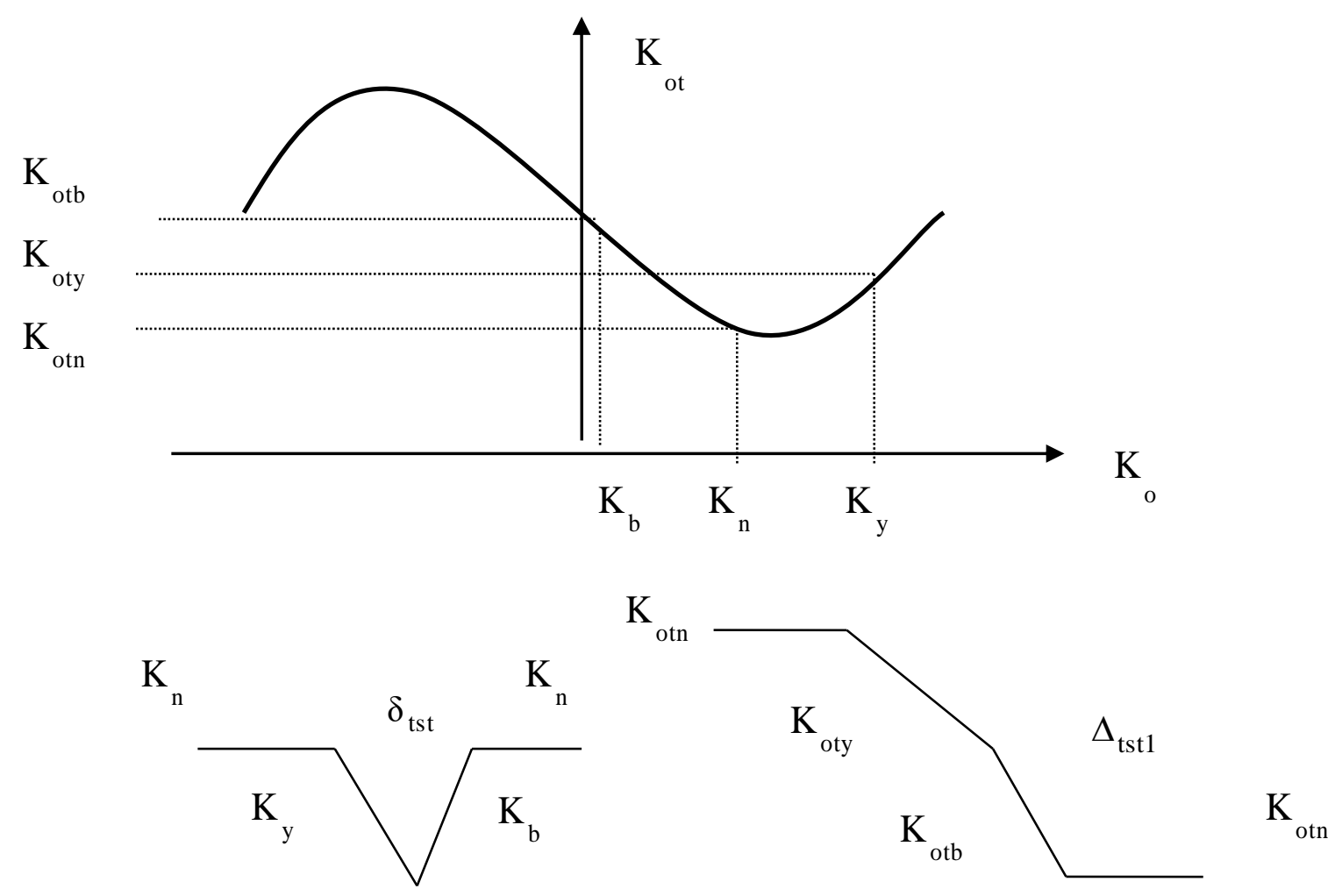

Рис. 6. Соответствие $\delta_{\mathrm{tst}} \rightarrow \Delta_{\mathrm{tst} 1}$ форм траекторий при $\mathrm{V}_{\mathrm{o}}<\mathrm{V}_{\mathrm{c}}$ 
Аналогично для формы $\delta_{\text {tpr }}$ истинной траектории расхождения при нахождении курсов $\mathrm{K}_{\mathrm{n}}, \mathrm{K}_{\mathrm{y}}$ и $\mathrm{K}_{\mathrm{b}}$ на различных участках зависимости относительного курса (растущему или убывающему) существуют все четыре формы относительной траектории расхождения. Так на рис. 7 приведено соответствие $\delta_{\text {tpr }} \rightarrow \Delta_{\text {tpr }}$, когда формы одинаковые, а соответствие $\delta_{\text {tpr }} \rightarrow \Delta_{\text {tst }}$ форм траекторий иллюстрируется на рис. 8. Соответствие двойного изменения относительного курса влево $\delta_{\text {tpr }} \rightarrow \Delta_{\text {tpr1 }}$ показано на рис. 9, а на рис. 10 приведено соответствие форм $\delta_{\text {tpr }} \rightarrow \Delta_{\text {tst1 }}$ двойного изменения относительного курса вправо.

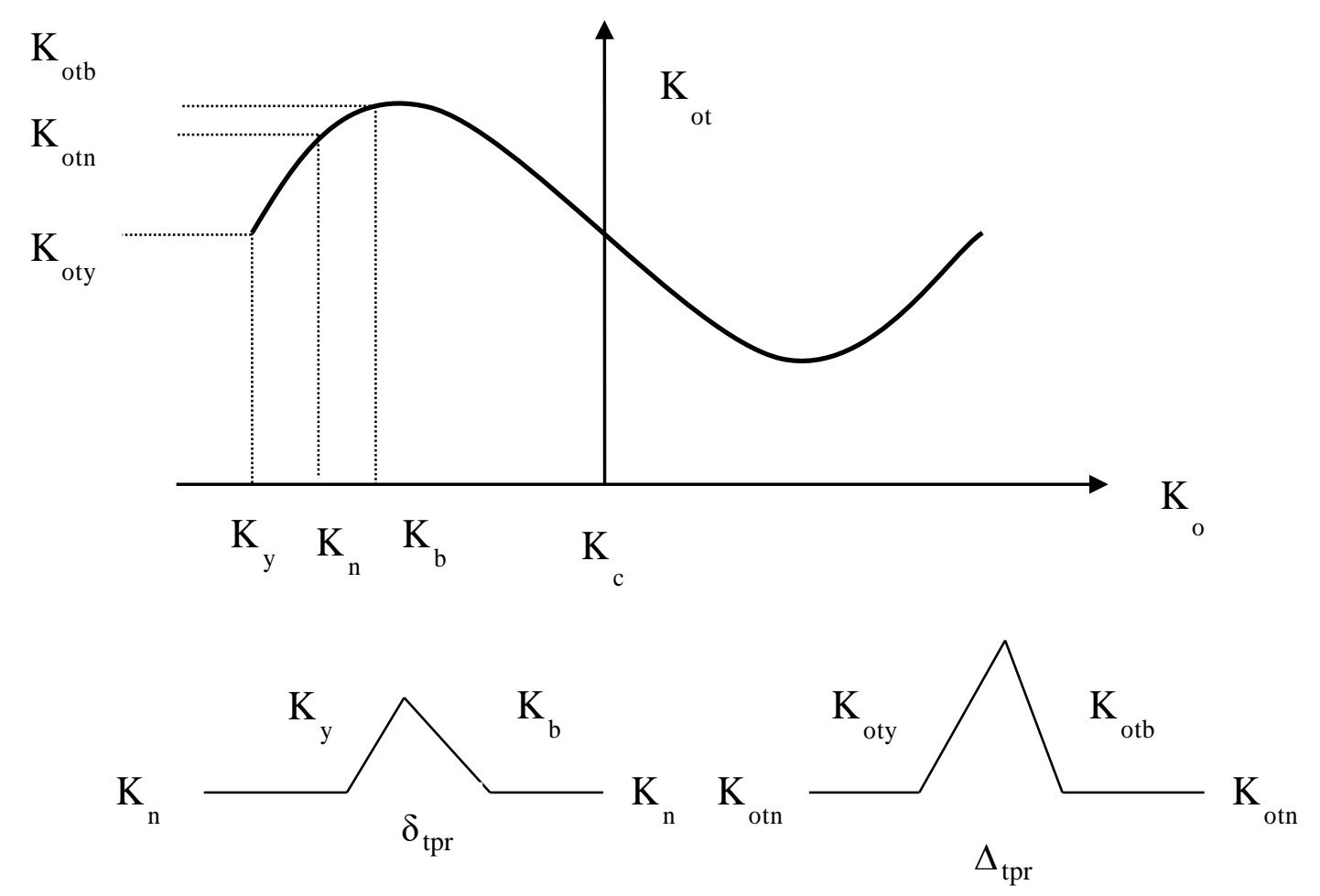

Рис. 7. Соответствие $\delta_{\mathrm{tpr}} \rightarrow \Delta_{\mathrm{tpr}}$ форм траекторий при $\mathrm{V}_{\mathrm{o}}<\mathrm{V}_{\mathrm{c}}$ 

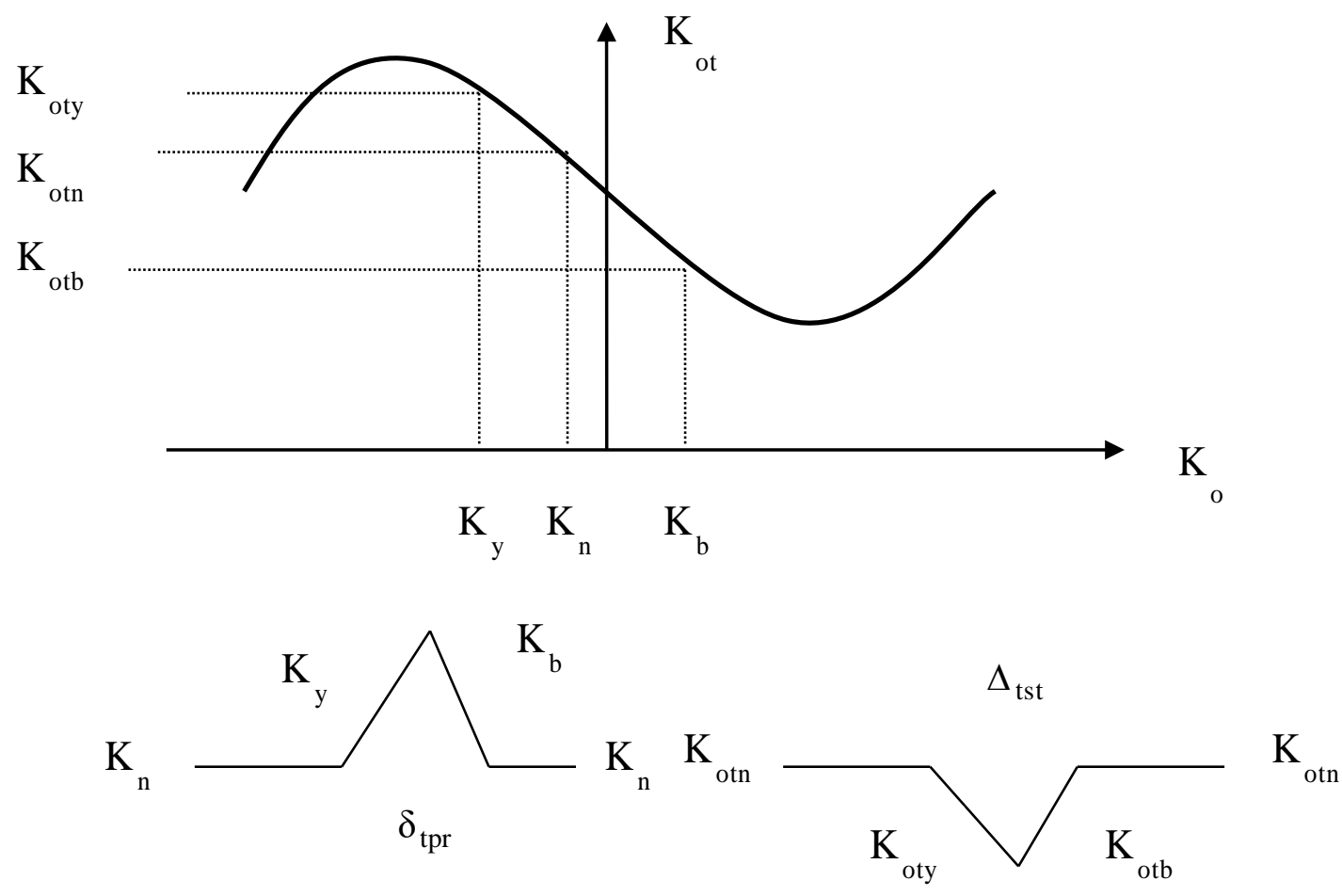

Pис. 8. Соответствие $\delta_{\text {tpr }} \rightarrow \Delta_{\text {tst }}$ форм траекторий при $\mathrm{V}_{\mathrm{o}}<\mathrm{V}_{\mathrm{c}}$

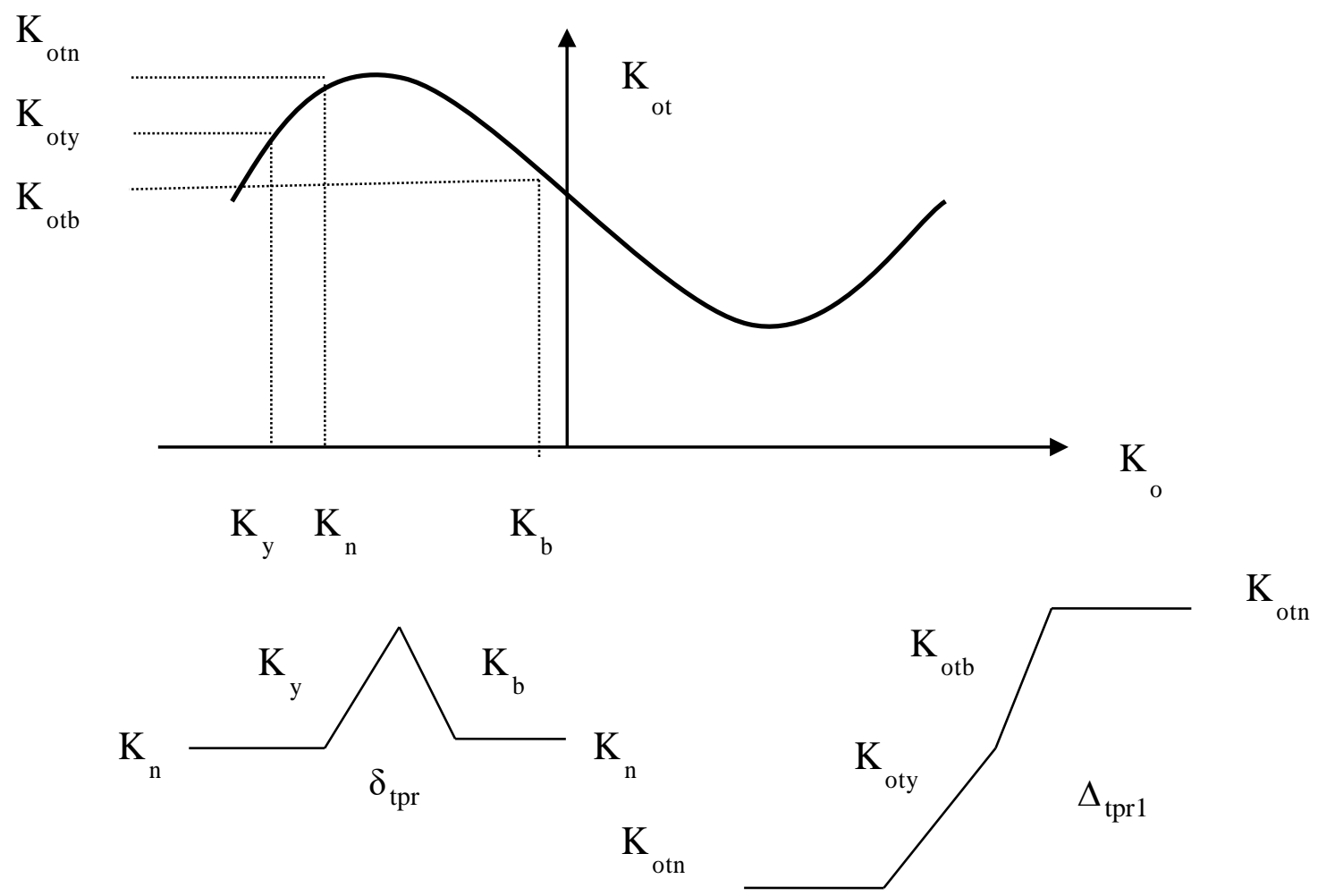

Puс. 9. Соответствие $\delta_{\mathrm{tpr}} \rightarrow \Delta_{\mathrm{tpr} 1}$ форм траекторий при $\mathrm{V}_{\mathrm{o}}<\mathrm{V}_{\mathrm{c}}$ 

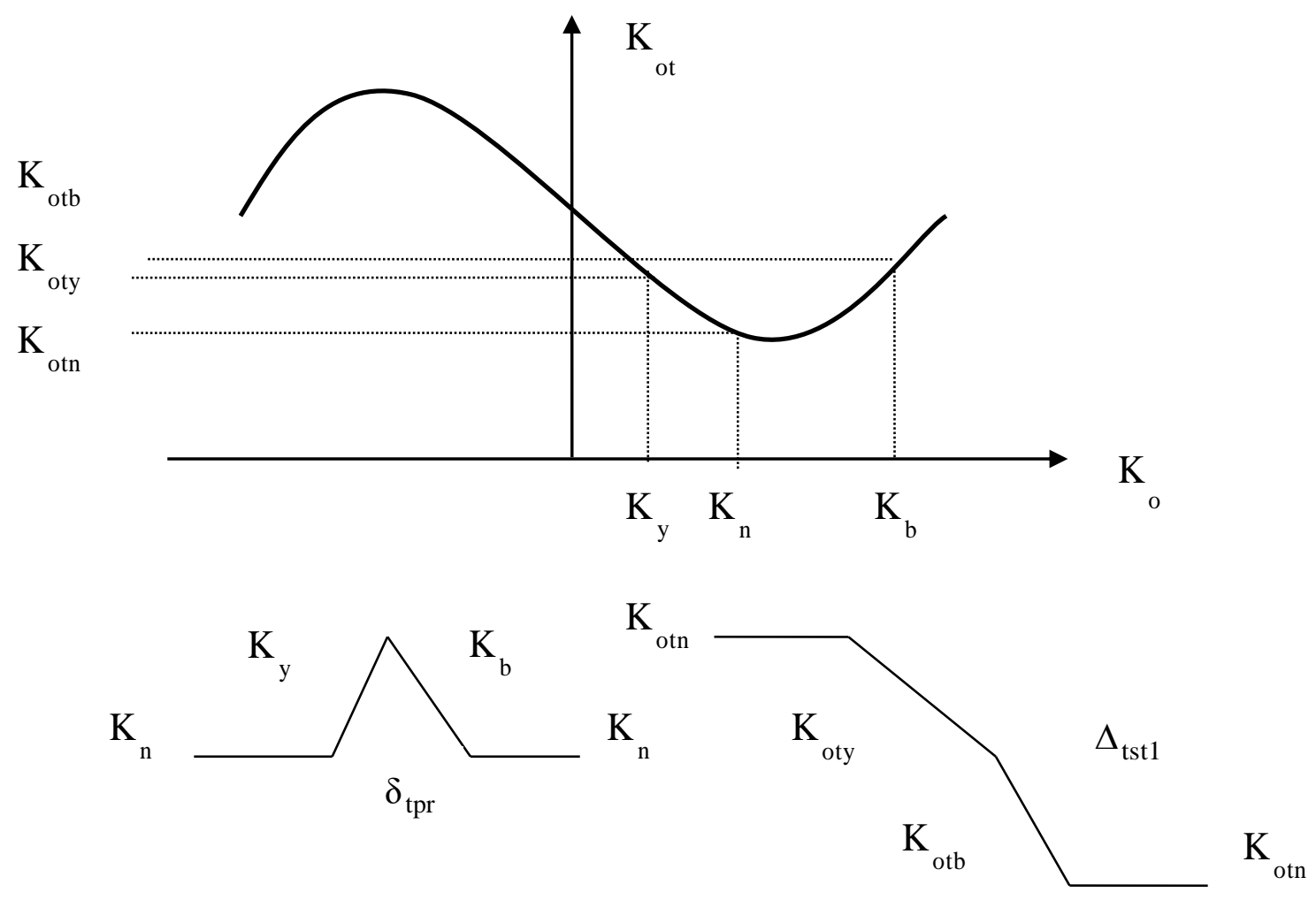

Рис. 10. Соответствие $\delta_{\mathrm{tpr}} \rightarrow \Delta_{\mathrm{tst} 1}$ форм траекторий при $\mathrm{V}_{\mathrm{o}}<\mathrm{V}_{\mathrm{c}}$

\section{Выводы и перспектива дальнейшей работы по данному направлению}

Таким образом, в общем случае формы $\delta_{\text {tst }}$ и $\delta_{\text {tpr }}$ истинной траектории расхождения могут отображаться в пространстве относительного движения четырьмя формами относительной траектории расхождения $\Delta_{\mathrm{tst}}, \Delta_{\mathrm{tpr}}, \Delta_{\mathrm{tst} 1}$ и $\Delta_{\mathrm{tpr1}}$. В дальнейшем целесообразно рассмотреть зависимость форм относительной траектории расхождения от отношения скорости судна к скорости цели.

\section{ЛИТЕРАТУРА}

1. Цымбал Н.Н. Гибкие стратегии расхождения судов / Цымбал Н.Н., Бурмака И.А., Тюпиков Е.Е. - Одесса: КП ОГТ, 2007. - 424 с.

2. Бурмака И.А. Управление судами в ситуации опасного сближения / И.А Бурмака., Э.Н Пятаков., А.Ю. Булгаков - LAP LAMBERT Academic Publishing, - Саарбрюккен (Германия), -2016. - 585 c.

3. Мальцев А. С. Учет маневренных характеристик для обеспечения безопасности плавания / Мальцев А. С. // Судостроение и ремонт. - 1989. №5. - C. 29-31. 
4. Мальцев А. С. Маневрирование судов при расхождении / Мальцев А.С. Одесса: Морской тренажерный центр, 2002. - 208 с.

5. Бурмака, И.А. Экстренная стратегия расхождения при чрезмерном сближении судов / Бурмака И.А., Бурмака А.И., Бужбецкий P.Ю. - LAP LAMBERT Academic Publishing, - Саарбрюккен (Германия), 2014. - 202 c. 